\title{
Chemical characterization by gas chromatography-mass spectrometry and inductively coupled plasma-optical emission spectroscopy of membrane permeates from an industrial dairy ingredient production used as process water
}

\author{
Peter B. Skou, ${ }^{* 1}$ Bekzod Khakimov, ${ }^{*}$ Thomas H. Hansen,† Stina D. Aunsbjerg, ${ }^{*}$ Susanne Knøchel,, \\ Dorrit Thaysen, $\ddagger$ and Frans van den Berg* \\ *Department of Food Science, and \\ †Department of Plant and Environmental Sciences, Faculty of Science, University of Copenhagen, Rolighedsvej 26, \\ DK-1958 Frederiksberg C, Denmark \\ ‡Arla Foods Ingredients, Sønderupvej 26, DK-6920 Videbæk, Denmark
}

\begin{abstract}
Reusing reverse osmosis (RO) membrane permeate instead of potable water in the dairy industry is a very appealing tactic. However, to ensure safe use, the quality of reclaimed water must be guaranteed. To do this, qualitative and quantitative information about which compounds permeate the membranes must be established. In the present study, we provide a detailed characterization of ultrafiltration, $\mathrm{RO}$, and $\mathrm{RO}$ polisher (ROP) permeate with regard to organic and inorganic compounds. Results indicate that smaller molecules and elements (such as phosphate, but mainly urea and boron) pass the membrane, and a small set of larger molecules (long-chain fatty acids, glycerol-phosphate, and glutamic acid) are found as well, though in minute concentrations $(<0.2 \mu M)$. Growth experiments with 2 urease-positive microorganisms, isolated from $\mathrm{RO}$ permeate, showed that the nutrient content in the ROP permeate supports limited growth of 1 of the 2 isolates, indicating that the ROP permeate may not be guaranteed to be stable during protracted storage.

Key words: dairy ingredient production, process water, membrane filtration, quality characterization
\end{abstract}

\section{INTRODUCTION}

Reducing the overall water footprint has become an important objective in the dairy processing industry due to increasing cost of discharge and intake as well as limited availability of potable water. The potable water consumption can be greatly reduced through efficient use of process waters, such as membrane permeate and

Received March 29, 2017.

Accepted August 24, 2017.

${ }^{1}$ Corresponding author: peter.b.skou@food.ku.dk evaporator condensate, in different areas of the production including cleaning processes.

In dairy processing facilities, membrane filtration technologies are already frequently used to create new products. These technologies can be characterized by their ability to separate molecules and constituents of different sizes. At Arla Foods Ingredients, Viby, Denmark, whey, a by-product from cheese production facilities, is processed into new products. First, UF membranes are used to retain whey proteins, and the permeate is processed through a 2-stage reverse osmosis (RO plus RO polisher; ROP) membrane plant to collect lactose. The collected whey proteins are used downstream to produce various products and constituents, whereas the lactose is used as a food ingredient (e.g., in infant formula). The focus of this investigation is the use potential of the ROP permeate as a process water stream.

Although use of process water is an attractive option, microbiological safety is of major importance in the food and dairy industry and must be ensured at all times. To do this, real-time online monitoring of relevant parameters should be considered (Casani et al., 2005). Process analytical technology provides the principles for real-time online monitoring (van den Berg et al., 2013), but to select the appropriate measurement technique the target molecule(s) must first be identified. The $\mathrm{RO}(\mathrm{P})$ permeates from dairy-derived water have been reported to have a very low organic load and low conductivity indicating minimal concentrations of organic compounds and minerals. Organic load has traditionally been expressed by classical, cumulative numbers such as total organic carbon, chemical oxygen demand, and total nitrogen (Vourch et al., 2005, 2008). However, these cumulative measurements do not provide insight into which specific compounds permeate the membranes. Vourch et al. (2008) looked at selected 


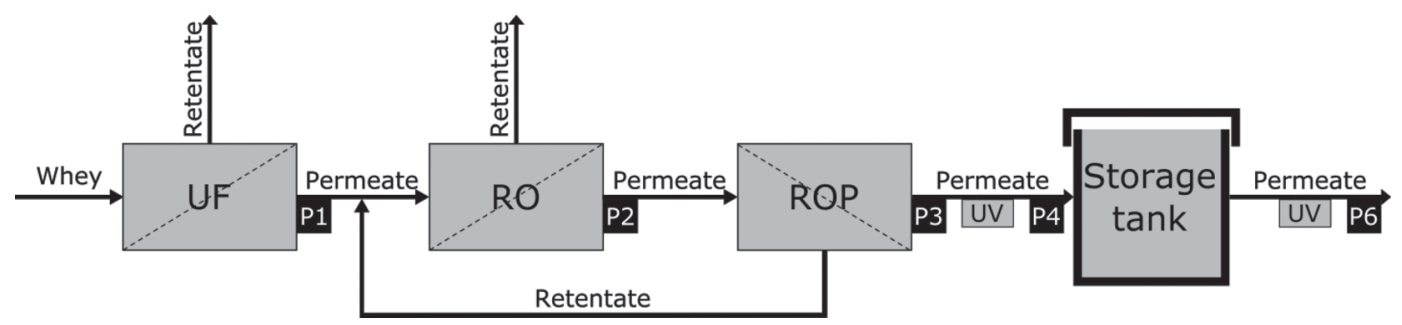

Figure 1. Schematic overview of UF processing and reverse osmosis (RO) plus RO polisher (ROP) lactose and water recovery plus storage. The process water sampling points are marked P1 to P6.

organic compounds and ions in membrane permeates and vapor condensates. A different approach is taken (e.g., in the field of metabolomics) where analytical techniques for the characterization of complex mixture samples have been developed. Through derivatization of functional groups with a labile proton using (e.g., trimethylsilyl), classes of compounds previously unsuitable for GC analysis become volatile and can be detected using fully automated sample preparation and analysis workflows (Khakimov et al., 2013). These analytical techniques open up for a 2-stage data analysis approach in the same analytical run: exploratory untargeted (unbiased) data analysis followed by a targeted analysis of a selection of compounds using a large sample set. These very low concentration compounds can be determined via a combination of automated sample pretreatment, GC, sensitive MS, and advanced data analysis. Regarding element analysis, inductively coupled plasma optical emission spectroscopy (ICPOES) has become more popular in recent years, providing a high-throughput, broad (unbiased) coverage both in terms of elements and concentration ranges (Hansen et al., 2009; Husted et al., 2011).

In the present study we characterize the chemical composition of UF, RO, ROP permeate, and storage tank water in a dairy ingredient production through untargeted derivatization based GC-MS coupled with advanced chemometric analysis. A selection of the chemical compounds are quantified via calibration standard series. Furthermore, detailed element composition of the streams is determined over a 10 -h production run through ICP-OES analysis to investigate the dynamic behavior of element retention. This information was supplemented with 6-d growth experiments (radically surpassing normal process water storage) performed with 2 microorganisms isolated from RO permeate, a Pseudomonas sp. and a Staphylococcus sp., to test if the low nutrient levels in the ROP permeate were sufficient to support microbial growth. To the best of our knowledge this is the first study performed for an untargeted chemical characterization and element analysis of dairy membrane permeates.

\section{MATERIALS AND METHODS}

\section{Samples Collected}

All samples were collected at the Arla Foods Ingredients production facilities (Nr. Vium, Denmark) in 250-mL amber, sterile, polypropylene sample bottles (Isolab, Wertheim, Germany) over a sample collection period of less than $1 \mathrm{~min}$, and stored at $5^{\circ} \mathrm{C}$ until analysis. Immediately before sampling, the process valves were opened and flushed to waste for approximately $10 \mathrm{~s}$. Conductivity was measured on site, directly after collection, and before analysis for quality assurance purposes. A schematic illustration of the process and sampling locations is presented in Figure 1. Samples are denoted as follows: P1, UF permeate; P2, RO permeate; P3, ROP permeate before UV treatment (400 $\mathrm{J} / \mathrm{m}^{2}, \mathrm{BX} 100 \mathrm{e}$, Wedeco, USA); P4, ROP permeate after UV; P6, ROP permeate after storage tank, and UV treatment.

Process water samples for GC-MS analysis were collected 3 or 4 times on the same day and analyzed in duplicate or triplicate for P1, P2, P3, P4, and P6, leading to 15 samples overall (and 46 analysis runs in total). Process water samples for ICP-OES were collected simultaneously from UF, RO, and ROP permeate (P1, $\mathrm{P} 2$, and $\mathrm{P} 4$, respectively; $40 \times 3$ permeate samples; for further details, see Skou et al., 2017a). Sampling was initiated immediately after the treatment plant was started (following a cleaning in place) and continued for approximately $10 \mathrm{~h}$. This time period included 2 feed tank changes where samples were collected with a higher frequency, accompanied by a lower sampling frequency in between.

The 2-stage $\mathrm{RO}+\mathrm{ROP}$ plant consists of the RO section with 9 loops in parallel each with 6 membrane elements in series and the ROP segment of 4 loops in parallel each with 6 membrane elements in series; all elements are DOW Hypershell RO-8038 (TetraPak, Silkeborg, Denmark). The feed to the RO+ROP plant is UF permeate from whey processing adjusted to $\mathrm{pH}$ 5.8 with a flow ranging from 100 to $150 \mathrm{~m}^{3} \cdot \mathrm{h}^{-1}$, target 
retentate concentration is $15^{\circ}$ Brix $(\sim 13.5 \% \mathrm{DM})$. It should be noted that samples taken from the different extraction points at the same time are representative for averages and trends, but a sample-by-sample comparison between locations is not feasible due to different volumes and residence times at the various stages in the treatment plant.

\section{Gas Chromatography-Mass Spectrometry}

For P1 samples, a 100- $\mu \mathrm{L}$ aliquot was dried in a 200$\mu \mathrm{L}$ glass insert using a ScanVac (LaboGene, Lynge, Denmark). For all other sample points a 1-mL aliquot was dried in a new glass vial using the ScanVac. The residues in the glass vials were re-suspended in $120 \mu \mathrm{L}$ of Milli-Q water and a $100-\mu \mathrm{L}$ aliquot out of this was further dried in $200-\mu \mathrm{L}$ glass inserts. After drying, the inserts were capped in GC vials before derivatization and GC-MS injection. Dilution series of authentic standards were prepared in a concentration range of 64.9 to $0.4125 \mu M$ for lactose, glucose, and galactose, and 32.5 to $0.2125 \mu M$ for oleic acid $(\mathrm{C} 18: 1)$ and linoleic acid (C18:2). Thirty microliters of each standard solution was completely dried in the ScanVac and prepared for the GC-MS analyses as described previously. The different and distinct volumes for sample and standard preparation steps (dilution factors) are corrected for in the reported results.

Dried samples were derivatized by the addition of $30 \mu \mathrm{L}$ of derivatization reagent trimethylsilyl cyanide (TMSCN, Fluka, Steinheim am Albuch, Germany) and agitation at $750 \mathrm{rpm}$ for $50 \mathrm{~min}$ as described earlier in literature (Khakimov et al., 2013). The GC-MS analysis was performed using an Agilent 7890B GC (Agilent Technologies, Santa Clara, CA) coupled with a HT Pegasus time-of-flight mass spectrometer (Leco Corporation, USA). The GC-MS data acquisition parameters were published previously in Khakimov et al. (2013). Some modifications were introduced in this study including the GC oven temperature gradient and MS settings: the initial temperature of the oven was $40^{\circ} \mathrm{C}$, held for $2 \mathrm{~min}$, heated to $220^{\circ} \mathrm{C}$ at a rate of $20^{\circ} \mathrm{C} \cdot \mathrm{min}^{-1}$, followed by $10^{\circ} \mathrm{C} \cdot \mathrm{min}^{-1}$ to reach a final temperature of $320^{\circ} \mathrm{C}$ at which the oven was kept for 8 min. The mass spectra were recorded in the range 45 to $500 \mathrm{~m} / z$ at a data acquisition rate of 10 spectra $\cdot \mathrm{s}^{-1}$. The raw GC-MS data were exported in the netCDF file format. The GC-MS data were processed using the freeware program PARADISe developed in our research group (www.models.life.ku.dk $\backslash$ PARADISe, accessed February 2017). This software enables deconvolution of peaks by means of PARAFAC2 (Johnsen et al., 2017). The PARAFAC2 (PF2) deconvoluted mass spectra were compared against the NIST11 GC-MS database version 2.0 [National Institute of Standards and Technology (NIST), Gaithersburg, MD]. The PF2 scores of deconvoluted peaks were extracted and used for subsequent data analysis. Scores from PF2 represent the normalized area of deconvoluted mass spectra, or in other words, the scores represent the normalized area of the specific compound extracted (Johnsen et al., 2017). All compounds were normalized with the score of a derivatization-agent-derived stable peak to correct for small differences in injection volume. Limit of detection (LOD) was set as a score value above the mean of the blanks plus 5 times the standard deviation of the blanks. If a specific compound was not included in a standard, that standard also served as blank for this compound, jointly with the 3 analytical blanks.

\section{Inductively Coupled Plasma-Optical Emission Spectroscopy}

Before analysis samples were acidified to $3.5 \% \mathrm{HNO}_{3}$ using $70 \% \mathrm{HNO}_{3}$ acid (Plasma-Pure, SCP Science, Marktoberdorf, Germany) to ensure fast washout inbetween samples. Multi-elemental analysis was hereafter performed on a 5100 ICP-OES (Agilent Technologies) equipped with a Meinhard nebulizer and a cyclonic spray chamber. For each sample 22 elements were measured simultaneously. A 10-point external calibration standard from CPI International (Amsterdam, the Netherlands) was also included. The P1 samples were measured undiluted, and $10 \times$ and $100 \times$ diluted, whereas all other samples were measured undiluted. It is known that carbon in the samples will affect the plasma and change the plasma temperature. This changes how and when elements are ionized and eventually excited in the plasma (Husted et al., 2011). For this reason samples are typically digested before analysis to decrease the carbon to a negligible amount. However, this is a time-consuming process that can be circumvented if matrix matching is possible or if it can be shown that the matrix does not affect the analysis. To evaluate the actual interference from carbon, in our case almost exclusively lactose, the 10-point calibration curve was mixed with increasing levels of lactose $(0,0.04,0.40$, and $4.00 \%$ wt/wt). After each 20 (randomized) samples, a set of analyses consisting of a blank, a drift sample, and a blank were run to ensure that no drift or carry-over would affect the subsequent measurements (Olsen et al., 2016). The drift sample used was a certified biological reference material (NIST 1515 , USA), certified for the elements presented in the Results section. 
From the available ICP-OES spectral lines, one was chosen for each element, using sensitivity to lactose and drift over the analysis run as selection criteria. Calibrations were built based on the most appropriate standards (i.e., those standards that covered the sample's expected concentration range as best as possible). While predicting the concentrations in $\mathrm{P} 1$, the dilution with the signal best covered by the calibrations was used. The limits of quantification (LOQ) were established on 37 blank samples, calculated as the mean of the blanks plus 10 times the standard deviation of the blanks for each element. All observations below the quantification limits were excluded from further data evaluation.

To quantify the migration behavior of the different elements in the membrane systems the rejection factor $(\mathrm{RF})$ was used:

$$
\mathrm{RF}=\left(1-\frac{\mathrm{C}_{\text {permeate }}}{\mathrm{C}_{\text {feed }}}\right) \cdot 100 \%
$$

where $\mathrm{C}_{\text {feed }}$ and $\mathrm{C}_{\text {permeate }}$ denote the concentration for the feed and permeate, respectively.

\section{Microbial Growth Potential}

Microorganisms were isolated from UF and RO permeate by spreading permeates on plate count agar and water plate count agar and incubating at 22 and $37^{\circ} \mathrm{C}$, respectively. Selected isolates were grown in urea broth $\left(0.1 \mathrm{~g} \cdot \mathrm{L}^{-1}\right.$ of yeast extract, $9.1 \mathrm{~g} \cdot \mathrm{L}^{-1}$ of $\mathrm{KH}_{2} \mathrm{PO}_{4}, 9.5$ $\mathrm{g} \cdot \mathrm{L}^{-1}$ of $\mathrm{Na}_{2} \mathrm{HPO}_{4}, 20 \mathrm{~g} \cdot \mathrm{L}^{-1}$ of urea, and $0.01 \mathrm{~g} \cdot \mathrm{L}^{-1}$ of phenol red, Merck, Kenilworth, NJ) at $30^{\circ} \mathrm{C}$ to test their ability to degrade urea (data not shown). Two urease-positive isolates were chosen to test their growth potential in ROP permeate. The isolates were identified by $16 \mathrm{~S}$ rDNA sequence analysis to be Pseudomonas sp. and Staphylococcus sp., respectively. The growth potential in ROP permeate was studied by inoculating cells $\left(10^{5} \mathrm{cfu} \cdot \mathrm{mL}^{-1}\right)$ washed with sterile physiological saline in sterile filtrated ROP permeate (P3 in Figure 1) and calculating $\mathrm{cfu} \cdot \mathrm{mL}^{-1}$ during storage at $16^{\circ} \mathrm{C}$ for up to $6 \mathrm{~d}$. The temperature $16^{\circ} \mathrm{C}$ was chosen because it is close to the temperature of the permeate during processing and the process water during storage, and thus the most relevant when investigating safety.

\section{Statistical Analysis}

Data were analyzed in Matlab R2015b (The MathWorks Inc., Natick, MA) using in-house routines.

\section{RESULTS}

\section{Gas Chromatography-Mass Spectrometry}

Compounds detected in the permeate samples are presented in Table 1, and some raw chromatograms of representative samples are shown in Figure 2. Table 1 illustrates at which sample point(s) the compounds were detected above the LOD. Detection $(+)$ was marked if at least one of the analyses from the 3 distinct sample replicates' collected for each sample point contained the compound above the LOD. Compound identification was categorized into 3 groups: level 1 identification was based on spectral similarity and retention time comparison with pure standards, level 2 was based on spectral similarity $>800$ and a Kovats index (van Den Dool and Kratz, 1963) within less than 30 units from the reported values in the NIST library, and level 3 was based only on spectral similarity $>800$ (Sumner et al., 2007).

The semi-quantification of the different samples based on peak area represented by PARAFAC2 scores for $\alpha$-ketoglutarate $\left(\mathrm{C}_{5} \mathrm{H}_{6} \mathrm{O}_{5}\right.$, also known as $\alpha$-ketoglutaric acid in the protonated form) is shown as an example in Figure 3. The standards and blanks show a relative concentration consistently close to score zero and P1 shows the highest values, accompanied by a relative large sample-to-sample variance. The most abundant peak by far in the GC-MS profiles for P2, P3, P4, and P6 samples corresponded to urea. This chemical compound has been quantified in this production process in a previous study (Skou et al., 2017a) and was omitted from the GC-MS interpretation due to severe peak overloading making it unsuitable for quantification.

All calibration curves developed for the selected compounds had squared Pearson correlation coefficients of $\mathrm{r}^{2}=0.88$ or higher. The lowest concentration of standards used for calibration curves are shown in Table 1. It was possible to quantify glucose $\left(\mathrm{r}^{2}=0.95\right)$ in $\mathrm{P} 1$, and lactose $\left(\mathrm{r}^{2}=0.94\right)$ and galactose $\left(\mathrm{r}^{2}=0.95\right)$ in $\mathrm{P} 2$. Glucose concentration in $\mathrm{P} 1$ samples was estimated to be between 0.01 and $0.06 \mathrm{mM}$, whereas lactose and galactose concentrations were estimated to be $0.02-0.10$ $\mathrm{m} M$ and $0.005-0.02 \mathrm{~m} M$, respectively, in P2 samples (Figure 4). Lactose and galactose concentrations in P1 samples were above the highest concentration standard $(0.0649 \mathrm{mM})$ used in this study, whereas all other samples/sampling locations were below the LOD. The concentration of linoleic acid in the process water samples was above LOD and close to the lowest concentration standard. Oleic acid was not found above the LOD in any process samples. 


\section{Inductively Coupled Plasma-Optical Emission Spectroscopy}

Calibration models for ICP-OES were tested for lactose interference (relevant especially for P1 samples), drift and linearity in the response range relevant for the samples. The final ICP-OES quantifications are presented in Table 2 as averages for each sampling point together with the number of measurements below the quantification limit, the average rejection factor over the RO and ROP membranes and the LOQ associated with the calibration for each observed element. As a point of reference the element composition of NIST Reference Material 1640a-Trace Elements in Natural Water is also included in Table 2.

Figure 5a presents the concentration of zinc as an example of the dynamic behavior and variance during the observed production run. The rejection for zinc per time point is presented in Figure 5b, and the averages of the 2 time series are included in Table 2.

To emphasize the connectivity between the elements found in process water samples, the correlation matrix, including measured conductivity, can be found in Table
3 ; the covariation between magnesium and conductivity as an example case is shown in Figure 6. Conductivity was on average reduced by $91.2 \%$ going from P1 to P2 and $82.2 \%$ going from P2 to P4.

\section{Microbial Growth Potential}

Figure 7 shows the microbial growth potential, indicating that Pseudomonas sp. was able to grow in ROP permeate, whereas Staphylococcus sp. was not. A log increase within $2 \mathrm{~d}$ was observed for Pseudomonas sp.

\section{DISCUSSION}

\section{Gas Chromatography-Mass Spectrometry}

Our GC-MS data analysis approach included (I) an exploratory untargeted approach where several dairy associated compounds were found (Table 1), and (II) a targeted quantification of selected compounds (Figure 4). With this approach, potential target molecules could be identified in P1, thereby making analysis of process water further along the process more focused

Table 1. Chemical compounds detected in process water samples arranged according to functional group ${ }^{1}$

\begin{tabular}{|c|c|c|c|c|c|c|c|c|c|}
\hline No. & Compound $^{2}$ & $\mathrm{KI} \mathrm{NIST}^{3}$ & KI exp. ${ }^{3}$ & $\mathrm{P} 1$ & $\mathrm{P} 2$ & P3 & $\mathrm{P} 4$ & $\mathrm{P} 6$ & $\begin{array}{c}\text { Lowest } \\
\text { concentration } \\
\text { standard }(\mu M)\end{array}$ \\
\hline 4 & L-Malic acid (2) & 1,538 & 1,516 & + & - & - & - & - & NA \\
\hline \multicolumn{10}{|c|}{ Sugars } \\
\hline 15 & Lactose (1) & 2,611 & 2,615 & + & + & - & - & - & 0.4125 \\
\hline 13 & Hexose (3) & $\sim 1,900$ & 1,979 & + & - & - & - & - & NA \\
\hline 8 & Pentose (3) & $\sim 1,750$ & 1,728 & + & - & - & - & - & NA \\
\hline \multicolumn{10}{|c|}{ Fatty acids } \\
\hline 14 & Linoleic acid (1) & 2,179 & 2,201 & + & - & - & - & - & 0.2125 \\
\hline-5 & Oleic acid (1) & 2,183 & - & - & - & - & - & - & 0.2125 \\
\hline 3 & Capric acid (2) & 1,455 & 1,485 & - & - & + & + & + & NA \\
\hline 7 & Lauric acid (2) & 1,651 & 1,673 & + & + & + & + & + & NA \\
\hline \multicolumn{10}{|c|}{ Miscellaneous } \\
\hline 1 & Phosphate (2) & 1,285 & 1,296 & + & + & + & + & + & NA \\
\hline$-^{5}$ & Urea (1) & - & - & + & + & + & + & + & NA \\
\hline
\end{tabular}

${ }^{1}$ Identification numbers correspond to peak numbers in Figure 2. $-=$ signals found to be less than the limit of detection (LOD; calculated as the mean of the blanks plus 5 times the SD of the blanks) for all samples; $+=$ signals found larger than LOD in at least one measurement from a given sampling point.

${ }^{2}$ Numbers in parentheses indicate $1=$ level 1 identification; $2=$ level 2 identification; $3=$ tentative identification.

${ }^{3}$ NIST $=$ National Institute of Standards and Technology. Kovats index (KI) based on direct injection of $1 \mu \mathrm{L}$ of alkane mixture (C10 to C40 from Sigma Aldrich, St. Louis, MO).

${ }^{4} \mathrm{NA}=$ not available.

${ }^{5}$ Compound not detected in process water samples. 
because compounds present in $\mathrm{P} 1$ are more likely to be present (in reduced concentrations) downstream.

In the initial exploratory analysis, compounds not included for targeted analysis were found (Table 1). Compounds that were not quantified using calibration curves are reported as either not detected (hence, below LOD) or detected (above LOD) only. Citric and malic acid are related to the primary energy metabolism of lactic acid bacteria in, for example, cheese making (Von Wright and Axelsson, 2012), whereas glycerol and glycerol-phosphate can be derived from either the primary energy metabolism or from fatty acid catabolism (Von Wright and Axelsson, 2012). L-Glutamic acid and its corresponding $\alpha$-keto-acid, $\alpha$-ketoglutarate, are important amino donors and receivers, respectively, in AA catabolism in cheese making and maturation (Ardö, 2006). The phosphate is likely from the phosphoric acid used to adjust $\mathrm{pH}$ before membrane filtration. Milk contains high amounts of the fatty acid triglycerides and free fatty acids are therefore expected to be present in the whey in low concentrations. Lactose and its breakdown products, glucose and galactose, constitute the primary content of the UF permeate and are thus anticipated at minor concentrations in downstream $\mathrm{RO}(\mathrm{P})$ permeates. To retain high signals for the low concentration compounds present in perme-
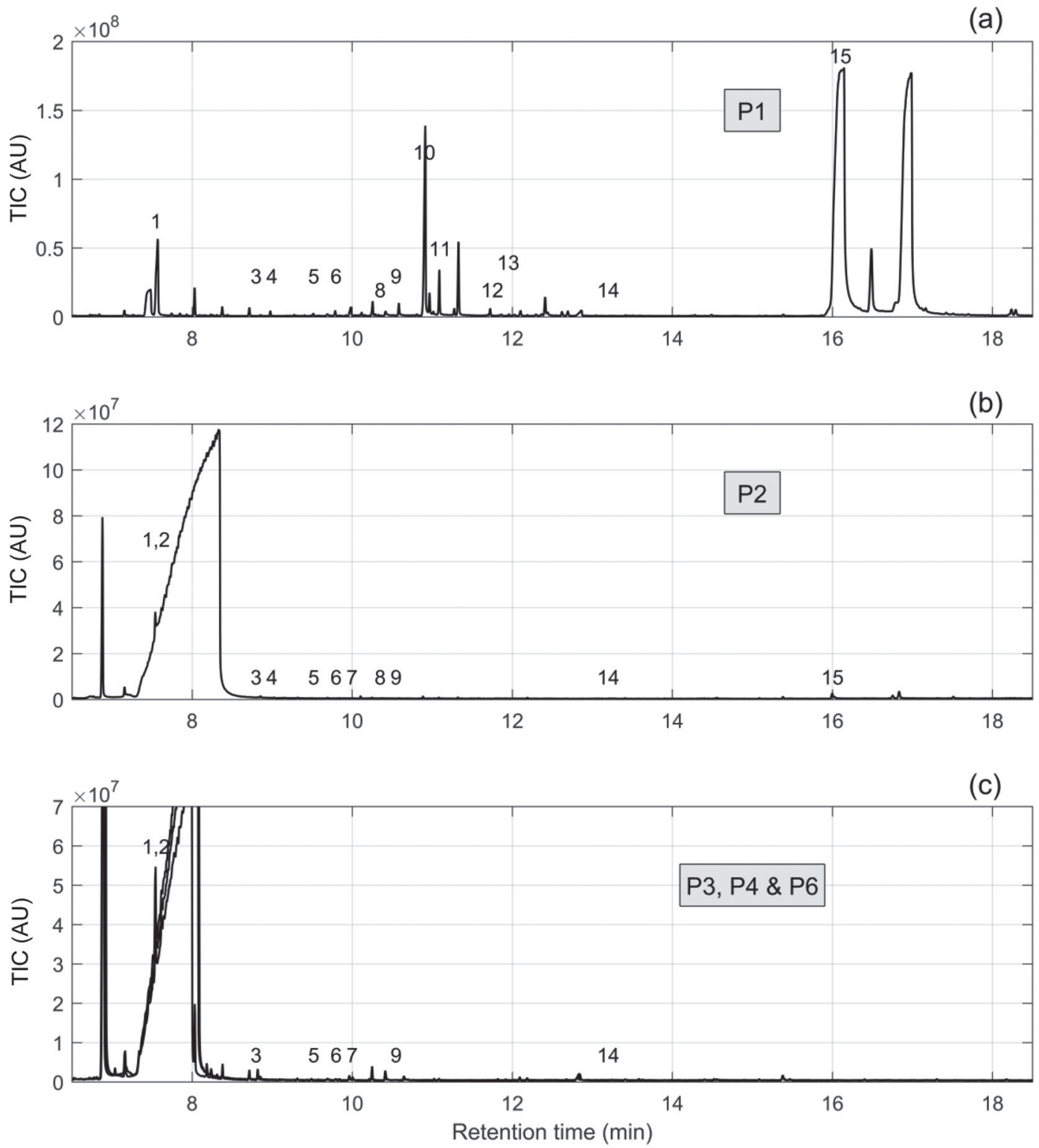

Figure 2. Examples of total ion count (TIC) chromatograms for single, representative (a) P1, (b) P2, and (c) P3, P4 plus P6 samples, with peak numbers corresponding to the compounds in Table 1 . The process water sampling points are marked P1 to P6. AU $=$ arbitrary units. 


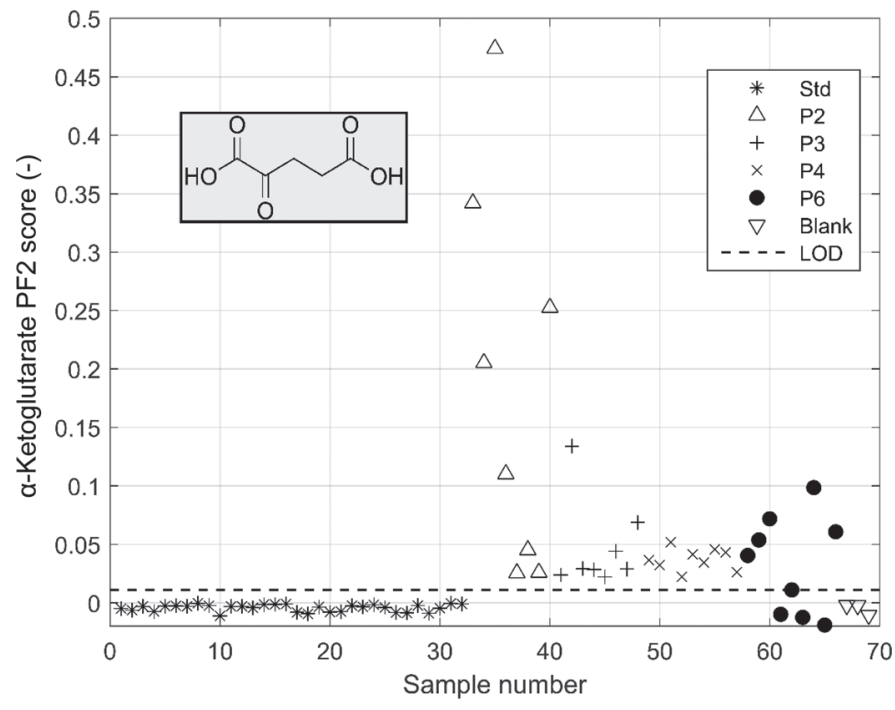

Figure 3. Relative concentration [PARAFAC2 (Johnsen et al., 2017; PF2) scores] of $\alpha$-ketoglutarate in all samples and standards. Relative concentrations of process water sampling point 1 (P1) samples (not shown) were between 1 and 9.2. LOD = limit of detection. Std $=$ standard .

ates, samples were trimethylsilylated directly, without prior methoximation. As a result, the unexpected peaks of hexose and pentose may derive from glucose and galactose (Koek et al., 2006). Without methoximation, several peaks corresponding to stereoisomers of carbohydrates (Evershed, 1993) make unambiguous identification almost impossible when complex samples are investigated. Urea has been known to pass RO membranes and the concentrations in the permeate using the present membrane processing system were reported earlier (Skou et al., 2017a). Glucose was quantified in P1 samples (0.01-0.06 $\mathrm{mM})$, whereas lactose and galac- tose were quantified in P2 samples $(0.02-0.10 \mathrm{mM}$ and 0.005-0.02 $\mathrm{m} M$, respectively).

When looking at Table 1, it is important to bear in mind that the samples collected simultaneously at points $\mathrm{P} 1$ to $\mathrm{P} 4$ (Figure 1) are not one-to-one comparable due to the large flows involved in parallel membrane batteries and the unknown hold-up times, whereas P6 samples are drawn from a storage tank. There will be an element of chance and intrinsic variability over time when sampling large industrial-scale systems, and one can only postulate that the samples collected are representative of the (average) process performance. Looking at the $\mathrm{RO}(\mathrm{P})$ permeates $(\mathrm{P} 3, \mathrm{P} 4$, and $\mathrm{P} 6)$, it was found that fatty acids, glycerol, AA, and phosphate pass the membrane to some extent. Interestingly, large molecules such as fatty acids seem to be able to pass the $\mathrm{RO}(\mathrm{P})$-membranes, albeit in low concentrations. This observation supports a recent study by CortésFrancisco and Caixach (2013) who investigated a seawater desalination process and also found fatty acids permeating the RO-membranes. It seems feasible that glycerol, the smallest AA glutamic acid and the corresponding $\alpha$-keto acid and phosphate, would be able to permeate the membranes in minute concentrations; however, to our knowledge no studies have reported this before.

Glycerol is detected in the $\mathrm{RO}(\mathrm{P})$ permeates, but not in the UF permeate, probably due to the higher concentration factor of the $\mathrm{RO}(\mathrm{P})$ samples. This could indicate that the glycerol concentration is more or less constant in the process water streams, suggesting that it can pass RO-membranes, as was also observed for urea (Skou et al., 2017a). However, this should be investigated further to get more solid confirmation. Phosphate was found in all process water streams. (a)

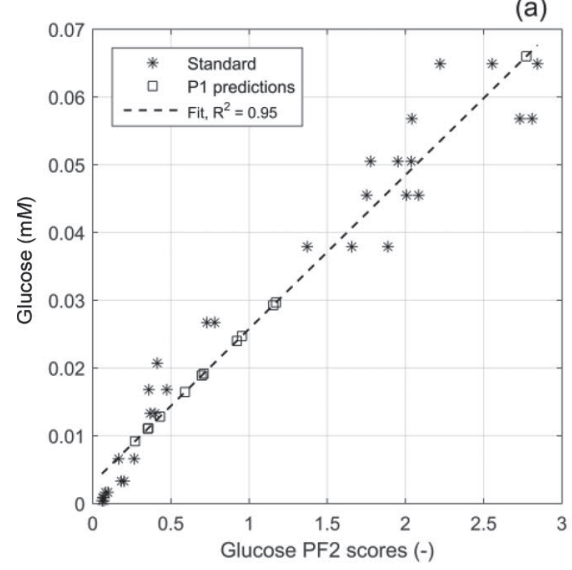

(b)

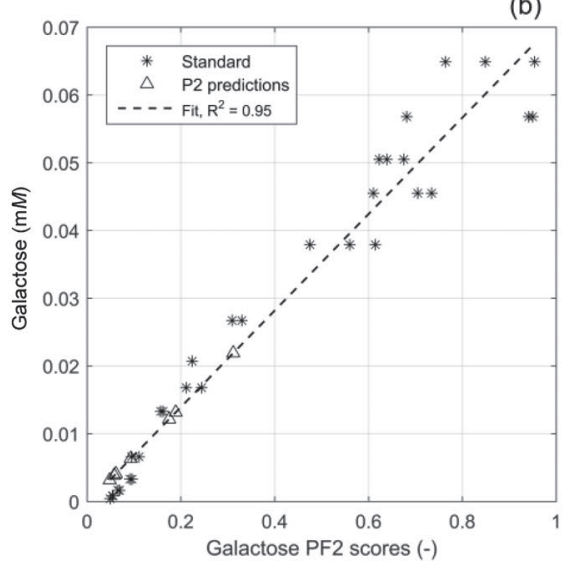

(c)

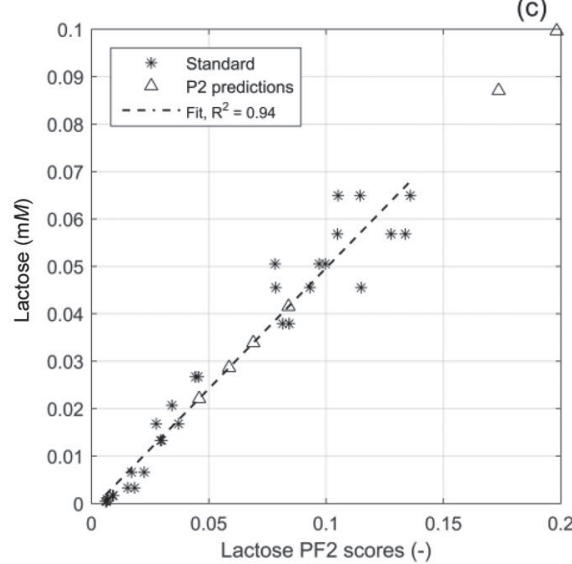

Figure 4. Quantification based on relative concentrations [PARAFAC2 (Johnsen et al., 2017; PF2) scores] and standard series for (a) glucose in process water sampling point 1 (P1) samples, and (b) galactose and (c) lactose in process water sampling point 2 (P2) samples. 
Table 2. Overview of elements detected in process water samples; the average concentration (based on samples with levels above the limit of quantification) in each process water sampling point; rejection factors over the reverse osmosis and reverse osmosis polisher membranes

\begin{tabular}{|c|c|c|c|c|c|c|c|c|c|}
\hline \multirow{2}{*}{$\frac{\text { Element }\left(\mathrm{LOQ}^{1}\right)}{\mathrm{Al}\left(2.7 \mu \mathrm{g} \cdot \mathrm{L}^{-1}\right)}$} & \multirow{2}{*}{$\begin{array}{c}\begin{array}{c}\text { NIST } \\
\text { ref. }\end{array} \\
53.0\end{array}$} & \multicolumn{6}{|c|}{$\begin{array}{c}\text { Concentration } \\
\left(\mu \mathrm{g} \cdot \mathrm{L}^{-1}\right)[\text { measurements below LOQ }]\end{array}$} & \multicolumn{2}{|c|}{ Rejection factor } \\
\hline & & \multicolumn{2}{|c|}{$\mathrm{P} 1[\mathrm{n}=40]$} & \multicolumn{2}{|c|}{$\mathrm{P} 2[\mathrm{n}=39]$} & \multicolumn{2}{|c|}{$\mathrm{P} 4[\mathrm{n}=40]$} & $\frac{\mathrm{P} 1 \text { to } \mathrm{P} 2}{82.3}$ & $\begin{array}{c}\mathrm{P} 2 \text { to } \mathrm{P} 4 \\
-\end{array}$ \\
\hline $\mathrm{B}\left(5.6 \mu \mathrm{g} \cdot \mathrm{L}^{-1}\right)$ & 303.1 & 92.7 & {$[0]$} & 65.0 & {$[0]$} & 38.1 & {$[0]$} & 29.2 & 41.3 \\
\hline $\mathrm{Ca}\left(25.0 \mu \mathrm{g} \cdot \mathrm{L}^{-1}\right)$ & $5.6 \cdot 10^{3}$ & $206 \cdot 10^{3}$ & {$[2]$} & 395 & {$[1]$} & - & {$[40]$} & 99.8 & - \\
\hline $\operatorname{Cr}\left(0.58 \mu \mathrm{g} \cdot \mathrm{L}^{-1}\right)$ & 40.5 & 6.34 & {$[37]$} & - & {$[39]$} & - & {$[40]$} & - & - \\
\hline $\mathrm{K}\left(2.42 \cdot 10^{3} \mu \mathrm{g} \cdot \mathrm{L}^{-1}\right)$ & 579.9 & $799 \cdot 10^{3}$ & {$[4]$} & $32 \cdot 10^{3}$ & {$[1]$} & $5.6 \cdot 10^{3}$ & {$[3]$} & 96.0 & 82.7 \\
\hline $\operatorname{Mg}\left(-1.1 \mu \mathrm{g} \cdot \mathrm{L}^{-1}\right)^{3}$ & $1.06 \cdot 10^{3}$ & $40.3 \cdot 10^{3}$ & {$[0]$} & 86.4 & {$[0]$} & 4.1 & {$[0]$} & 99.3 & 93.8 \\
\hline Mo $\left(1.6 \mu \mathrm{g} \cdot \mathrm{L}^{-1}\right)$ & 45.6 & 11.7 & {$[3]$} & - & [39] & - & {$[40]$} & - & - \\
\hline $\mathrm{Na}\left(200 \mu \mathrm{g} \cdot \mathrm{L}^{-1}\right)$ & $3.14 \cdot 10^{3}$ & $115 \cdot 10^{3}$ & 0 & $3.41 \cdot 10^{3}$ & {$[0]$} & 590 & {$[0]$} & 96.5 & 81.5 \\
\hline $\mathrm{P}\left(38.0 \mu \mathrm{g} \cdot \mathrm{L}^{-1}\right)$ & - & $232 \cdot 10^{3}$ & {$[0]$} & 614 & {$[1]$} & - & {$[40]$} & 99.4 & - \\
\hline $\mathrm{S}\left(110 \mu \mathrm{g} \cdot \mathrm{L}^{-1}\right)$ & - & $26 \cdot 10^{3}$ & {$[0]$} & 66 & {$[1]$} & 15.0 & {$[8]$} & 95.1 & 77.3 \\
\hline $\mathrm{Zn}\left(3.7 \mu \mathrm{g} \cdot \mathrm{L}^{-1}\right)$ & 55.6 & 33.9 & {$[0]$} & 8.7 & {$[1]$} & 8.2 & {$[0]$} & 70.0 & -0.6 \\
\hline
\end{tabular}

${ }^{1}$ Limits of quantification (LOQ) were calculated as the mean of the blanks plus 10 times the SD of the blanks for each element.

${ }^{2}$ National Institute of Standards and Technology (NIST) Standard Reference Material 1640a.

${ }^{3}$ Negative LOQ is a result of the large span in the standards causing the model to show a small bias in the very low concentration range.

Comparing this with the ICP-OES results, where P4 samples are below the detection limit $\left(38 \mu \mathrm{g} \cdot \mathrm{L}^{-1}\right)$, suggests that the concentration permeating is very low indeed. The compounds found in the ROP permeates were all in very low concentrations (except urea) and on the border of the detection limit. Nevertheless, the

(a)
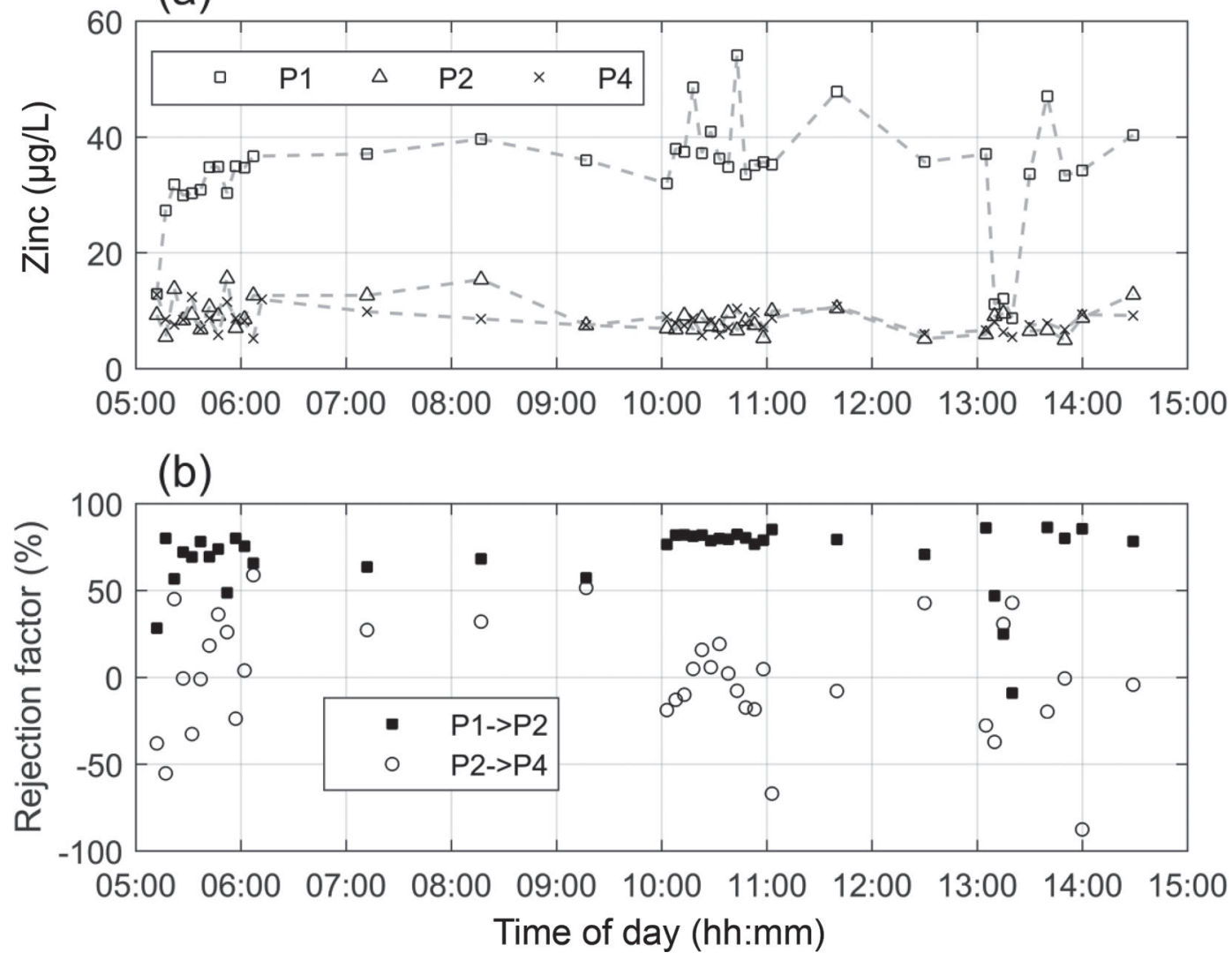

Figure 5. (a) Zinc concentration at the 3 sampling locations and (b) zinc rejection factor over a 10-h processing period. 
analysis does suggest that some compounds beside urea do permeate the ROP-membranes.

We attempted to perform unbiased GC-MS analysis and employed limited sample pre-cleaning and extraction. The GC-MS approach applied in this study was focused to detect compounds with molecular mass of up to $1 \mathrm{kDa}$. The compounds must also be volatile and thermally stable to be detected by our method. The derivatization usually enhances the above-mentioned 2 characteristics, enabling better detection. However, the drying stage involved in this protocol may result in evaporation of naturally volatile compounds such as ethanol and short-chain fatty acids.

\section{Inductively Coupled Plasma-Optical Emission Spectroscopy}

As described earlier, it is known that carbon in the samples will affect the plasma and change the plasma temperature. In this study we chose not to digest samples as this is a time-consuming process; instead, lactose matrix interference was tested. None of the spectral lines selected were found to be sensitive to lactose (carbon) at low levels $(0.04 \% \mathrm{wt} / \mathrm{wt})$.

From the total 22 elements available in the ICP-OES procedure, 13 elements were sensitive enough to quantify in the process water samples as presented in Table 2. Chrome, copper, iron, and molybdenum levels were so minimal that only very few samples were above the limit of quantification; this also means that rejection calculations could not be performed. For aluminum 7, P2 samples were above the detection limit and the rejection calculation was performed, which should, however, be interpreted with caution based on the small number of observations. Only 2 P4 samples were above the LOQ and the result is reported here only to illustrate the levels found in some process water samples. Our findings support the results from Vourch et al. (2008) for $\mathrm{Ca}^{2+}, \mathrm{Mg}^{2+}, \mathrm{Na}^{+}, \mathrm{K}^{+}$, and $\mathrm{HPO}_{4}^{+}$rejection in $\mathrm{RO}$ permeate, where we found $>99,>99,94-99,87-98$, and $>99 \%$, respectively. The negative detection limit for magnesium is an artifact of the calibration, which is focused on the expected range of the samples, leading to a poor estimation of the blank values. For zinc a $70 \%$ rejection is obtained from the first RO-membrane, but no additional reduction appears over the ROPmembrane. From Figure 5, the dynamics of the process becomes clear, especially around the tank change at 10:00 $\mathrm{h}$ where more extreme observations are made. This type of information could lead plant managers and optimization engineers to investigate extreme behavior of the process (Skou et al., 2017b). Sulfur is rejected effectively over both membrane plants, leading to a rejection efficiency of 95 and $77 \%$, respectively. Boron was detected in all samples (also as a consequence of the ICP-OES method being very sensitive toward this element) and results show a modest rejection efficiency of the $\mathrm{RO}(\mathrm{P})$-membranes (29.2 and $41.3 \%$, respectively). Similar rejection efficiencies have been reported by Rodríguez Pastor et al. (2001), who also hypothesize that the low efficiency is due to boron being in the form of boric acid, which has no ionic charge, making it permeate the membranes much like water. Despite the poor rejection, the concentrations found at 92.7, 65.0, and $38.1 \mu \mathrm{g} \cdot \mathrm{L}^{-1}$ (with an average SE across all predictions of $0.44 \mu \mathrm{g} \cdot \mathrm{L}^{-1}$ ) for UF, RO, and ROP permeate, respectively, are far below the maximum guideline values of $2.4 \cdot 10^{3} \mu \mathrm{g} \cdot \mathrm{L}^{-1}$ specified by the World Health Organization (WHO, 2009) for potable water, a conclusion that holds for all elements in the P3 stream. Aluminum was in a low concentration in $\mathrm{P} 1\left(17.2 \mu \mathrm{g} \cdot \mathrm{L}^{-1}\right)$ and was reduced close to the detection limit $\left(2.7 \mu \mathrm{g} \cdot \mathrm{L}^{-1}\right)$ for $\mathrm{P} 2$.

From the correlation matrix presented in Table 3, it can be seen that all the elements are highly correlated

Table 3. Squared Pearson correlation $\left(\mathrm{r}^{2}\right)$ between all elements and conductivity (Cond.) over all measurements; Cr, Cu, and Fe are not reported (-) due to less than $20 \%$ of the observations being available for calculation, as decided from the limits of quantification (calculated as the mean of the blanks plus 10 times the SD of the blanks for each element)

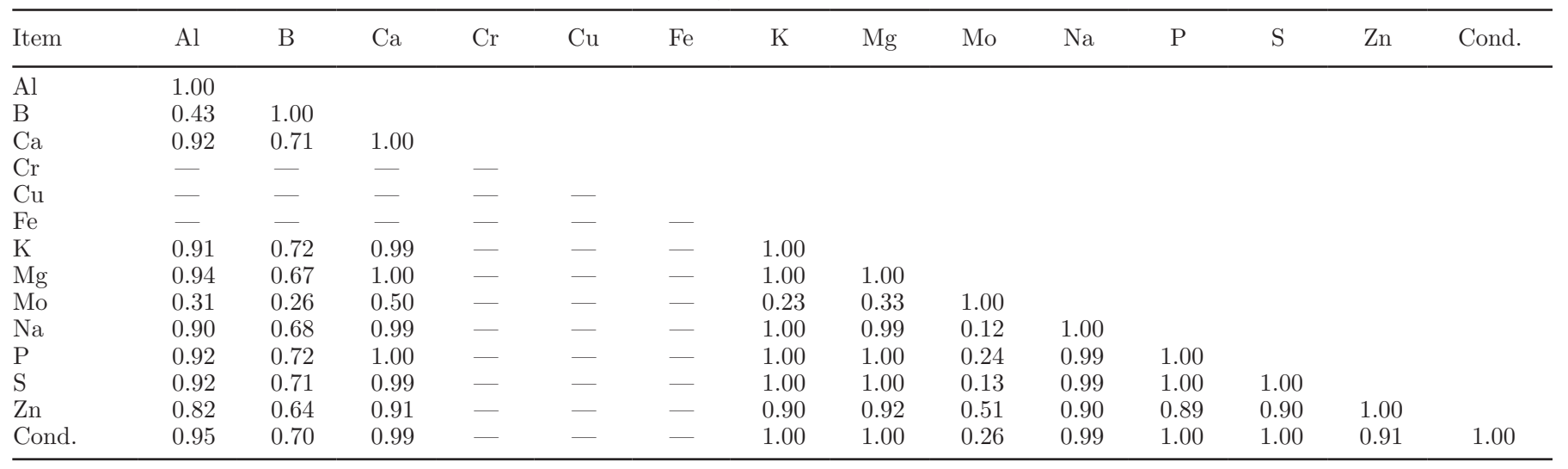



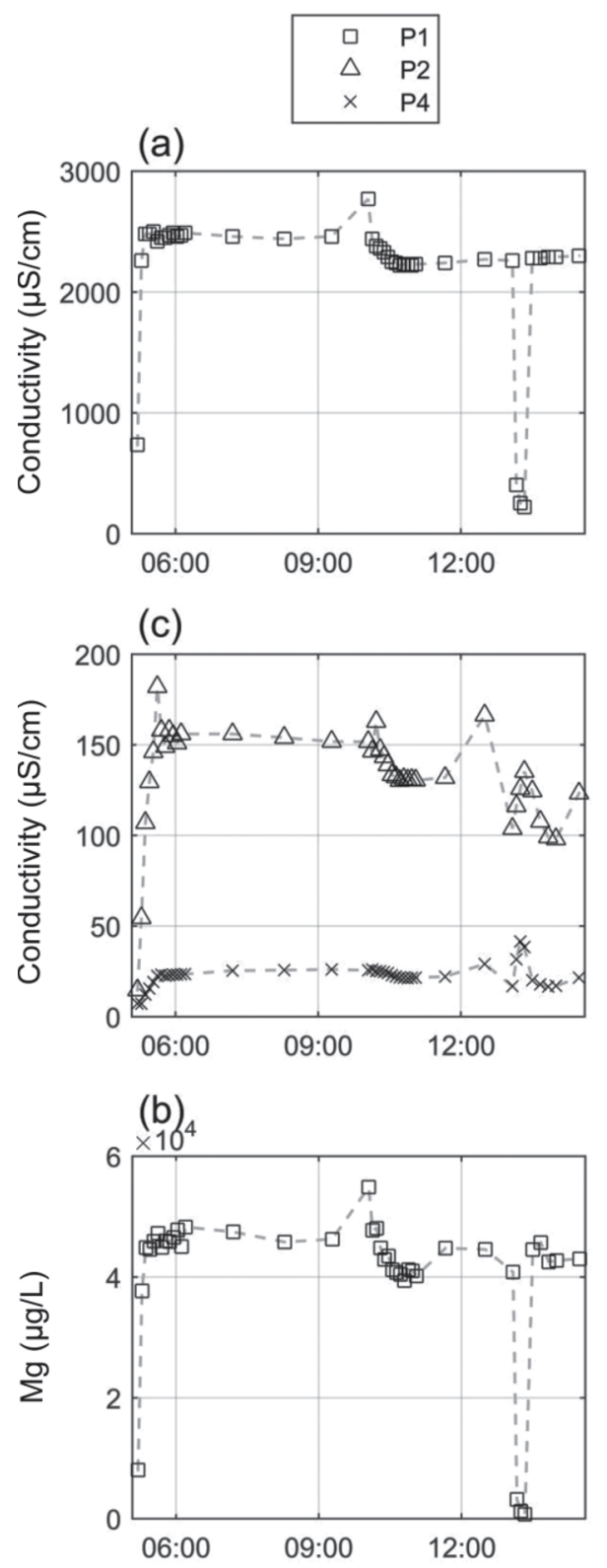

(d)

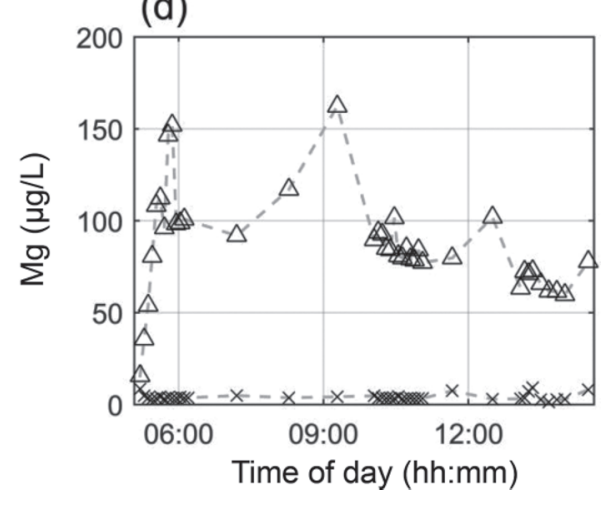

Figure 6. (a, c) Conductivity values and (b, d) magnesium concentration for all samples from sampling points $(\mathrm{a}, \mathrm{b}) \mathrm{P} 1$ and $(\mathrm{c}, \mathrm{d})$ $\mathrm{P} 2$ plus $\mathrm{P} 4$. The process water sampling points are marked $\mathrm{P} 1$ to $\mathrm{P} 4$. except for boron and molybdenum, where it should be reminded that the former is not rejected effectively and the latter was quantified only in a few measurements and these were close to the LOQ. Conductivity correlates strongly with all other elements. This suggests that conductivity can be safely used as an indirect measure of the element concentration, as is done in daily dairy operational practice. The constant rejection profiles over time in Figure 5 indicate that the process was running very stable, except during, for example, tank change, for the sampled period and that our findings are thus representative of normal operating conditions (Skou et al., 2017a).

\section{Microbial Growth Potential}

Although ROP permeate contains very low levels of bacteria, there is still a risk that undesirable growth may occur if some of the population is able to proliferate. Out of 2 urease positive strains, a Pseudomonas sp. and a Staphylococcus sp. isolated from RO permeate, only the Pseudomonas sp. was able to grow. The inoculation levels were several logs higher than found in any permeate and growth seemed to cease around $5 \times 10^{6}$ $\mathrm{cfu} \cdot \mathrm{mL}^{-1}$. The observations nevertheless indicate that even the very low nutrient ROP permeate may support growth at $16^{\circ} \mathrm{C}$ of part of the microbial population present. The Staphylococcus sp. survived but was not able to grow in the ROP permeate despite its urease activity, suggesting that additional nutrients were needed for the growth of this organism.

\section{CONCLUSIONS}

This investigation presents novel untargeted and targeted methodologies to characterize the chemical composition of process water derived from membrane processes in a dairy ingredient production facility. This first unbiased look into the chemical composition forms the input for a measurement-based safe use of reclaimed process water in the dairy industry. The methodologies presented facilitate further investigations potentially leading to periodic, high-sample-number risk assessment of process water and use potential to guarantee a hygienic and safe process operation. Results suggest that bigger compounds (long-chain fatty acids, glycerol-phosphate, and glutamic acid) do permeate the ROP-membranes but in very minute concentrations, whereas the elements boron and urea permeates readily. Although the nutrient levels were very low, Pseudomonas sp. originating from process water was able to grow in ROP permeate stressing the importance of controlling temperature and maximum-allowed storage times of the permeate intended for use. 


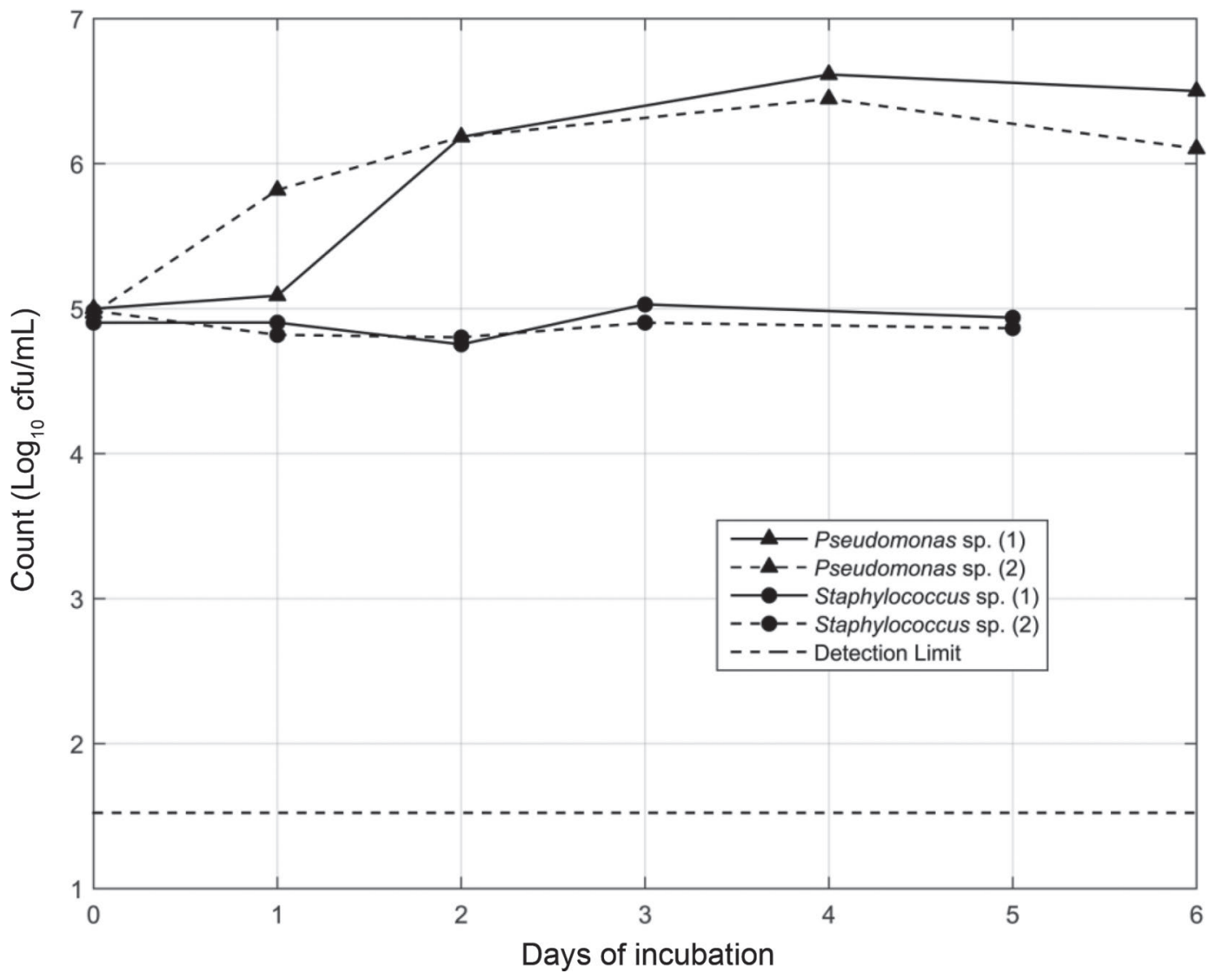

Figure 7. Growth curves of Pseudomonas sp. and Staphylococcus sp. in reverse osmosis polisher permeate at $16^{\circ} \mathrm{C}$.

\section{ACKNOWLEDGMENTS}

This work was supported by the REuse of WAteR in the food and bioprocessing inDustries (REWARD) consortium funded by the Danish Council for Strategic Research, Programme Commission on Health Food and Welfare (Copenhagen).

\section{REFERENCES}

Ardö, Y. 2006. Flavour formation by amino acid catabolism. Biotechnol. Adv. 24:238-242. https://doi.org/10.1016/j.biotechadv.2005 .11 .005 .

Casani, S., M. Rouhany, and S. Knøchel. 2005. A discussion paper on challenges and limitations to water reuse and hygiene in the food industry. Water Res. 39:1134-1146. https://doi.org/10.1016/ j.watres.2004.12.015.

Cortés-Francisco, N., and J. Caixach. 2013. Molecular characterization of dissolved organic matter through a desalination process by high resolution mass spectrometry. Environ. Sci. Technol. 47:96199627. https://doi.org/10.1021/es4000388.

Evershed, R. P. 1993. Advances in Silylation. K. Blau and J. M. Halket, ed. John Wiley \& Sons, Hoboken, NJ.

Hansen, T. H., K. H. Laursen, D. P. Persson, P. Pedas, S. Husted, and J. K. Schjoerring. 2009. Micro-scaled high-throughput digestion of plant tissue samples for multi-elemental analysis. Plant Methods 5: https://doi.org/10.1186/1746-4811-5-12.
Husted, S., D. P. Persson, K. H. Laursen, T. H. Hansen, P. Pedas, M. Schiller, J. N. Hegelund, and J. K. Schjoerring. 2011. Review: The role of atomic spectrometry in plant science. J. Anal. At. Spectrom. 26:52-79. https://doi.org/10.1039/C0JA00058B.

Johnsen, L. G., P. B. Skou, B. Khakimov, and R. Bro. 2017. Gas chromatography-mass spectrometry data processing made easy. J. Chromatogr. A 1503:57-64. https://doi.org/10.1016/j.chroma .2017 .04 .052 .

Khakimov, B., M. S. Motawia, S. Bak, and S. B. Engelsen. 2013. The use of trimethylsilyl cyanide derivatization for robust and broadspectrum high-throughput gas chromatography-mass spectrometry based metabolomics. Anal. Bioanal. Chem. 405:9193-9205. https://doi.org/10.1007/s00216-013-7341-z.

Koek, M., B. Muilwijk, M. J. van der Werf, and T. Hankemeier. 2006. Microbial metabolomics with gas chromatography/mass spectrometry. Anal. Chem. 78:1272-1281. https://doi.org/10.1021/ ac051683+.

Olsen, L. I., T. H. Hansen, C. Larue, J. T. Østerberg, R. D. Hoffmann, J. Liesche, U. Krämer, S. Surblé, S. Cadarsi, V. A. Samson, D. Grolimund, S. Husted, and M. Palmgren. 2016. Mother-plantmediated pumping of zinc into the developing seed. Nat. Plants 2:16036. https://doi.org/10.1038/nplants.2016.36.

Rodríguez Pastor, M., A. Ferrándiz Ruiz, M. F. Chillón, and D. Prats Rico. 2001. Influence of $\mathrm{pH}$ in the elimination of boron by means of reverse osmosis. Desalination 140:145-152. https://doi.org/10 .1016/S0011-9164(01)00364-2.

Skou, P. B., T. A. Berg, S. D. Aunsbjerg, D. Thaysen, M. A. Rasmussen, and F. van den Berg. 2017a. Monitoring process-water quality using near infrared spectroscopy and partial least squares regression with prediction uncertainty estimation. Appl. Spectrosc. 71:410-421. https://doi.org/10.1177/0003702816654165. 
Skou, P. B., S. E. Holroyd, and F. van den Berg. 2017b. Tutorial-Applying extreme value theory to characterize food-processing systems. J. Chemom. e2896. https://doi.org/10.1002/cem.2896.

Sumner, L. W., A. Amberg, D. Barrett, M. H. Beale, R. Beger, C. A. Daykin, T. W.-M. Fan, O. Fiehn, R. Goodacre, J. L. Griffin, T. Hankemeier, N. Hardy, J. Harnly, R. Higashi, J. Kopka, A. N. Lane, J. C. Lindon, P. Marriott, A. W. Nicholls, M. D. Reily, J. J. Thaden, and M. R. Viant. 2007. Proposed minimum reporting standards for chemical analysis Chemical Analysis Working Group (CAWG) Metabolomics Standards Initiative (MSI). Metabolomics 3:211-221. https://doi.org/10.1007/s11306-007-0082-2.

van den Berg, F., C. B. Lyndgaard, K. M. Sørensen, and S. B. Engelsen. 2013. Process Analytical Technology in the food industry. Trends Food Sci. Technol. 31:27-35. https://doi.org/10.1016/j.tifs 2012.04.007.

van Den Dool, H., and P. D. Kratz. 1963. A generalization of the retention index system including linear temperature programmed gas-liquid partition chromatography. J. Chromatogr. 11:463-471. https://doi.org/10.1016/S0021-9673(01)80947-X.

Von Wright, A., and L. Axelsson. 2012. Lactic Acid Bacteria: An Introduction. 4th ed. S. Lahtinen, A. C. Ouwehand, S. Salminen, and A. Von Wright, ed. CRC Press, Boca Raton, FL.

Vourch, M., B. Balannec, B. Chaufer, and G. Dorange. 2005. Nanofiltration and reverse osmosis of model process waters from the dairy industry to produce water for reuse. Desalination 172:245-256. https://doi.org/10.1016/j.desal.2004.07.038.

Vourch, M., B. Balannec, B. Chaufer, and G. Dorange. 2008. Treatment of dairy industry wastewater by reverse osmosis for water reuse. Desalination 219:190-202. https://doi.org/10.1016/j.desal 2007.05.013.

WHO. 2009. Boron in drinking-water. Background document for development of WHO Guidelines for Drinking-Water Quality. World Health Organization, Geneva, Switzerland. 